\title{
RECOVERING A FUNCTION FROM ITS GRAM DETERMINANTS ${ }^{1}$
}

\section{F. ALBERTO GRÜNBAUM}

Abstract. We prove that any real valued symmetric function defined on $A \times A, A$ an arbitrary set, can be determined up to a phase factor from the knowledge of its Gram determinants.

Let $X$ be an arbitrary set and $R$ a real valued function defined on $X \times X$, symmetric in the sense that $R(i, j)=R(j, i), i, j \in X$.

Assume that for each $n=1,2, \cdots$ and any choice of elements $i_{1}, \cdots, i_{n}$ in $X$, we know the value of the Gram determinants

$$
G_{R}\left(i_{1}, \cdots, i_{n}\right)=\operatorname{det} R\left(i_{s}, i_{r}\right), \quad s, r=1,2, \cdots, n .
$$

In this note we consider the problem of recovering the function $R$ from these determinants. We have previously considered this question in the case where the set $X$ is taken to be the real line and the function $R$ is assumed to be continuous and positive on the diagonal. See [1] and [2]. Under these extra requirements $R$ can be uniquely read off from these determinants, and it is not hard to see that the conditions are not only sufficient but (essentially) necessary for the uniqueness of $R$. Our main result follows.

THEOREM. If $R$ and $Q$ are a pair of real valued symmetric functions on $X \times X$ and for any $i_{1}, \cdots, i_{n}$ in $X$ one has $G_{R}\left(i_{1}, \cdots, i_{n}\right)=G_{Q}\left(i_{1}, \cdots, i_{n}\right)$, then

$$
R(i, j)=\phi(i) Q(i, j) \phi(j)
$$

for some function $\phi$ which takes only values \pm 1 .

REMARK. It follows directly from our proof that if $R$ and $Q$ are taken to be complex valued functions, or even functions taking values in any field, the same conclusion holds.

We chose to state the theorem in the real valued case because for the applications we envisage, the natural information is not the determinant

Received by the editors July 6, 1972 .

AMS (MOS) subject classifications (1970). Primary 41A63, 62H20, 60G15; Secondary 50B99.

Key words and phrases. Gram determinants.

${ }^{1}$ This research was supported by the National Science Foundation under grant NSF-GP-27719.

(c) American Mathematical Society 1974 
(1) but only its real part. Likewise the natural assumption on $R$ is $R(i, j)=$ $\mathrm{Cl}(R(j, i))$.

The proof is a consequence of the following two lemmas.

Lemma I. Let $R$ be a real valued, symmetric function defined on $X \times X$. Starting with all the determinants (1), one can compute any one of the expressions

$$
\sum_{\sigma} R\left(i_{\sigma 1}, i_{\sigma 2}\right) R\left(i_{\sigma 2}, i_{\sigma 3}\right) \cdots R\left(i_{\sigma n}, i_{\sigma 1}\right) .
$$

Here $\left(i_{1}, \cdots, i_{n}\right)$ is an arbitrary subset of $X$ and the sum extends to all permutations of $n$ elements.

Proof. The proof is carried out by induction on the number of points $i_{1}, \cdots, i_{n}$. For $n=1, G_{R}(i)=R(i, i)$ and there is nothing to prove. For $n=2$ we observe that $G_{R}(i, j)=R(i, i) R(j, j)-R(i, j) R(j, i)$, and now it is clear that

$$
\frac{1}{2} \sum_{\sigma} R\left(i_{\sigma 1}, i_{\sigma 2}\right)=G_{R}\left(i_{1}\right) G_{R}\left(i_{2}\right)-G_{R}\left(i_{1}, i_{2}\right) .
$$

Take as inductive the hypothesis that we can express (2) in terms of $G_{R}$ 's any time that we are dealing with less than $n$ points. Expand now the determinant $G_{R}\left(i_{1}, \cdots, i_{n}\right)$ in the customary way as a sum of different contributions corresponding to permutations of the set $1,2, \cdots, n$.

If one recalls that every permutation factors as a product of cycles, and that the signature sgn $\pi$ of a cycle of length $n$ is just $(-1)^{n-1}$, we obtain

$$
\begin{aligned}
G_{R}\left(i_{1}, \cdots, i_{n}\right) & =\sum_{\pi}(\operatorname{sgn} \pi) R\left(i_{1}, i_{\pi 1}\right) \cdots R\left(i_{n}, i_{\pi n}\right) \\
& =\left((-1)^{n-1} / 2\right) \sum_{\sigma} R\left(i_{\sigma 1}, i_{\sigma 2}\right) R\left(i_{\sigma 2}, i_{\sigma 3}\right) \cdots R\left(i_{\sigma n}, i_{\sigma 1}\right)+\sum_{\pi^{\prime}} .
\end{aligned}
$$

In the last line the first summand gives the contribution from those permutations $\pi$ which are cycles, and the second that of all the rest. But this remainder can be expressed as a sum of products of expressions like (2) involving fewer than $n$ arguments. For instance for $n=2$ we get from (3) the relation

$$
G_{R}\left(i_{1}, i_{2}\right)=-\frac{1}{2} \sum_{\sigma} R\left(i_{\sigma 1}, i_{\sigma 2}\right) R\left(i_{\sigma 2}, i_{\sigma 1}\right)+R\left(i_{1}, i_{1}\right) R\left(i_{2}, i_{2}\right) .
$$

By inductive hypothesis this remainder is thus known in terms of $G_{R}$ 's, and we conclude that (2) is expressible in terms of $G_{R}$ 's. The proof of the lemma is now complete.

We show now that each individual term in (2) can be read off. 
Lemma II. Let $i_{1}, \cdots, i_{n}$ be arbitrary points in $X$. The value of

$$
R\left(i_{1}, i_{2}\right) R\left(i_{2}, i_{3}\right) \cdots R\left(i_{n}, i_{1}\right)
$$

can be expressed in terms of expressions of the type (2) involving no more than $n$ arguments.

Proof. This proof is also done by induction on $n$. For $n=1$ we have nothing to prove. For $n=2,3$ the proof is trivial once we use the symmetry of the function $R$. Before taking up the general case we look into $n=4$.

Given $i_{1}, i_{2}, i_{3}, i_{4}$ we want to determine the value of

$$
R\left(i_{1}, i_{2}\right) R\left(i_{2}, i_{3}\right) R\left(i_{3}, i_{4}\right) R\left(i_{4}, i_{1}\right)
$$

in terms of expressions like those in (2) involving at most four arguments. The absolute value of each factor in (5) is known, and we can assume they are all nonzero, or else we already have (5). In this case (2) gives

$$
\begin{aligned}
& R\left(i_{1}, i_{2}\right) R\left(i_{2}, i_{3}\right) R\left(i_{3}, i_{4}\right) R\left(i_{4}, i_{1}\right)+R\left(i_{1}, i_{2}\right) R\left(i_{2}, i_{4}\right) R\left(i_{4}, i_{3}\right) R\left(i_{3}, i_{1}\right) \\
&+ R\left(i_{1}, i_{3}\right) R\left(i_{3}, i_{2}\right) R\left(i_{2}, i_{4}\right) R\left(i_{4}, i_{1}\right) .
\end{aligned}
$$

If we subtract from here the known terms

and

$$
\text { we get exactly (5). }
$$

$$
\frac{R\left(i_{1}, i_{2}\right) R\left(i_{2}, i_{4}\right) R\left(i_{4}, i_{1}\right) \times R\left(i_{1}, i_{4}\right) R\left(i_{4}, i_{3}\right) R\left(i_{3}, i_{1}\right)}{R\left(i_{4}, i_{1}\right) R\left(i_{1}, i_{4}\right)}
$$

Now to the general case, assuming as before that (4) is nonzero. In (2) each term is associated to a cycle involving the $n$ elements $i_{1}, \cdots, i_{n}$. We will see now that the contribution to (2) corresponding to any cycle which is not $\left(i_{1}, \cdots, i_{n}\right)$-or its inverse-can be expressed in terms of known factors, pretty much in the fashion of $(6)$ and $\left(6^{\prime}\right)$.

For any such cycle at least one of the adjacencies in $\left(i_{1}, i_{2}, \cdots, i_{n}\right)$ is broken, say that of $i_{p}$ and $i_{p+1}$. The cycle in question will look like $\left(i_{p}, i_{\alpha}, i_{\beta}, \cdots, i_{\gamma}, i_{p+1}, i_{\delta}, \cdots, i_{\varepsilon}\right)$, and its contribution to (2) is

$$
R\left(i_{p}, i_{\alpha}\right) R\left(i_{\alpha}, i_{\beta}\right) \cdots R\left(i_{\gamma}, i_{p+1}\right) R\left(i_{p+1}, i_{\delta}\right) \cdots R\left(i_{\varepsilon}, i_{p}\right) .
$$

Now this can be rewritten as

$$
\frac{R\left(i_{p}, i_{\alpha}\right) \cdots R\left(i_{\gamma}, i_{p+1}\right) R\left(i_{p+1}, i_{p}\right) \times R\left(i_{p}, i_{p+1}\right) R\left(i_{p+1}, i_{\delta}\right) \cdots R\left(i_{\varepsilon}, i_{p}\right)}{R\left(i_{p+1}, i_{p}\right) R\left(i_{p}, i_{p+1}\right)}
$$


and each one of the two factors in the numerator as well as the denominator is known by inductive hypothesis. Clearly, the same argument goes through if $i_{p+1}$ occurs before $i_{p}$, and the lemma is proved.

ProOF OF THE THEOREM. From the assumption, $G_{R} \equiv G_{Q}$, and the lemmas above we conclude that for any choice of elements $i_{1}, \cdots, i_{n}$ one has

$$
R\left(i_{1}, i_{2}\right) R\left(i_{2}, i_{3}\right) \cdots R\left(i_{n}, i_{1}\right)=Q\left(i_{1}, i_{2}\right) Q\left(i_{2}, i_{3}\right) \cdots Q\left(i_{n}, i_{1}\right) .
$$

Pick a pair of indices $(i, j)$ such that $R(i, j) \neq 0$. Fix $j$, set $\phi(j)=1$, and for every $i$ such that $R(i, j) \neq 0$, define $\phi(i)$ to be

$$
\phi(i)=\frac{Q(i, j)}{R(i, j)} .
$$

To extend the definition of $\phi$ to the whole of $X$ we proceed by exhaustion. Fix an $i$ of those involved in (8), and for every index $k$ on which $\phi$ is as yet undefined and satisfies $R(i, k) \neq 0$, define

$$
\phi(k)=\frac{Q(i, k)}{R(i, k)} \phi(i) .
$$

If $X$ is not exhausted by now, pick a different $i^{\prime}$ appearing in (8) and define $\phi$ on all those $k^{\prime}$ satisfying $R\left(i^{\prime}, k^{\prime}\right) \neq 0$, and previously out of reach, to be

$$
\phi\left(k^{\prime}\right)=\frac{Q\left(i^{\prime}, k^{\prime}\right)}{R\left(i^{\prime}, k^{\prime}\right)} \phi\left(i^{\prime}\right) .
$$

We keep going in this way, employing if necessary some $k$ appearing in (9) and defining $\phi(l)$, if as yet undefined and $R(l, k) \neq 0$, to be

$$
\phi(l)=\frac{Q(k, l)}{R(k, l)} \phi(k)
$$

If we continue in this fashion, we exhaust all of $X$ with the exception of a set of values $U$, such that it is impossible to construct a chain from any one of them to $j$, such that at each step in the chain the value of $R$ between two consecutive points is nonzero. Now, either $R$ vanishes if any of its arguments is in this set $U$, or else we can take some point in $U$ as a new starting point as above, define $\phi$ to be unity there, and proceed as before, extending $\phi$ to some points in $U$, and so on. It is clear now that we eventually can define $\phi$ on the whole of $X$.

It remains to show that, for any pair $i, j$, we have

$$
R(i, j)=\phi(i) Q(i, j) \phi(j)
$$


Given a pair $(i, j)$, either they have a "common ancestor", that is to say, there exists an index $k$, and a pair of chains $(k, \alpha, \beta, \cdots, \varepsilon, i),(k, \gamma$, $\delta, \cdots, v, j)$ such that

and

$$
R(k, \alpha) R(\alpha, \beta) \cdots R(\varepsilon, i) \neq 0,
$$

$$
R(k, \gamma) R(\gamma, \delta) \cdots R(\nu, j) \neq 0,
$$

or else they come from different ancestors $k, k^{\prime}$. Because of the exhaustive method of construction, the second case can arise only if $R(i, j)=Q(i, j)=$ 0 , and in this case we have nothing to prove. In the first case, if one brings in the definitions of $\phi(i), \phi(\varepsilon), \cdots, \phi(\alpha)$ and $\phi(j), \phi(v), \cdots, \phi(\gamma)$, see (8) and (9), we get

and

$$
\phi(i)=\frac{Q(i, \varepsilon)}{R(i, \varepsilon)} \cdots \frac{Q(\alpha, k)}{R(\alpha, k)}
$$

$$
\phi(j)=\frac{Q(j, v)}{R(j, v)} \cdots \frac{Q(\gamma, k)}{R(\gamma, k)} .
$$

Thus checking (10) amounts to proving

$$
\begin{aligned}
Q(i, \varepsilon) \cdots Q(\alpha, k) Q(i, j) Q(j, v) & \cdots Q(\gamma, k) \\
= & R(i, j) R(i, \varepsilon) \cdots R(\alpha, k) R(j, v) \cdots R(\gamma, k) .
\end{aligned}
$$

Using the symmetry of $R$ and $Q$, this is an immediate consequence of (7). The proof of the Theorem is finished.

Applications. (I) Let $z(i), i \in X$, denote a Gaussian family of real valued random variables. Take the means to be zero and the variances one. It is not hard to see that the knowledge of the finite-dimensional distributions of the family

$$
w(i)=z^{2}(i), \quad i \in X,
$$

is equivalent to the knowledge of the quantities (2) introduced above, provided we set $R(i, j)=E(z(i) z(j))$. See for instance [1].

Suppose now that two Gaussian families $z(i)$ and $z^{\prime}(i)$ give the same finite-dimensional distributions once they are squared as in (11).

Our theorem above says that this implies

$$
\begin{aligned}
E(z(i) z(j)) & =R(i, j)=\phi(i) R^{\prime}(i, j) \phi(j)=\phi(i) E\left(z^{\prime}(i) z^{\prime}(j)\right) \phi(j) \\
& =E\left(\phi(i) z^{\prime}(i) \phi(j) z^{\prime}(j)\right) .
\end{aligned}
$$

We can summarize this by saying that it all looks as if we had $z(i)=$ $\phi(i) z^{\prime}(i), i \in X$, for a nonrandom function $\phi$. For the case when $R$ is continuous the reader can consult [1]. 
(II) Let $u(i)$ and $v(i)$ denote a pair of vector valued functions in a real Hilbert space $H$.

It is not hard to see that a partial isometry $O$ satisfying $O u(i)=v(i)$ exists if and only if

$$
\langle u(i), u(j)\rangle=\langle v(i), v(j)\rangle, \quad i, j \in X .
$$

Our theorem above says that if for any choice of $i_{1}, \cdots, i_{n}$, the volume of the parallelograms generated by the vectors $u\left(i_{1}\right) \cdots u\left(i_{n}\right)$ and $v\left(i_{1}\right) \cdots$ $v\left(i_{n}\right)$ respectively, are equal, then one has

$$
\begin{aligned}
\langle u(i), u(j)\rangle & =\phi(i)\langle v(i), v(j)\rangle \phi(j) \\
& =\langle\phi(i) v(i), \phi(j) v(j)\rangle .
\end{aligned}
$$

This can be summed up by saying that the equality of the corresponding volumes implies that $u(i)$ is isometric to $\phi(i) v(i)$ for some appropriate $\phi$ taking only values \pm 1 . For the case when the curves are taken to be continuous, and avoiding the origin in $H$, the reader can consult [2].

\section{BIBLIOGRAPHY}

1. F. Alberto Grünbaum, The square of a Gaussian process, Z. Wahrscheinlichkeitstheorie und Verw. Gebiete 23 (1972), 121-124.

2. - The orthogonal invariants of a curve in Hilbert space, Proc. Amer. Math. Soc. (to appear).

Department of Mathematics, Courant Institute of Mathematical Sciences, New York UnIVERSITY, NeW York, NeW York 10012

Current address: Department of Applied Mathematics, California Institute of Technology, Pasadena, California 91109 